\title{
Gender Classification and Age Estimation using Neural Networks: A Survey
}

\author{
Gangesh Trivedi \\ School of Computer Engineering and Technology, \\ MIT World Peace University, \\ Pune, Maharashtra, India
}

\author{
Nitin N. Pise, PhD \\ School of Computer Engineering and Technology, \\ MIT World Peace University, \\ Pune, Maharashtra, India
}

\begin{abstract}
Researchers have shown more interest in soft biometrics area to fill the commination gaps between humans and machines with the growth of real-world application has increased day to day life. Soft-biometric consists of age, gender, ethnicity, height, facial measurements and etc. This paper contains a detail discussion about the contribution of the researchers in the area of gender classification and age estimation using neural networking. Most of the work is done using Convolutional neural networks and auto encoders. Various elements related to neural network model such as dataset, findings, calculative metrics and results are embraced for effortless interpretation of tabular correlation research. Finally, the authors summarize germane tasks for future various research aspects.
\end{abstract}

\section{Keywords}

Soft Biometrics, Neural Nets, CNN, Gender recognition, Age estimation

\section{INTRODUCTION}

A Human face provides a lot of information about the age, gender, mood etc. It is affected by many dynamic factors that get changed over period of time such as aging, hair style, expressions, etc. Gender and Age are considered an important biometric attribute for human identification. Bio-metric recognition is the method of gathering information about person's physiological and behavioural characteristics for human identification and verification (security models). Biometrics consists of soft biometric (age, gender, ethnicity, height and facial measurements) and hard biometric (Physical, behavioural and biological). Soft-biometric attributes like skin, hair colour, distance between eye and nose, face shape, and etc can be accessed to accelerate data traversing, or to classify unlabelled subject for various gender and age classes.

Furthermore, with the widespread use of computers, biometric identification comes into demand in areas such as home automation and healthcare. Recently, it has come about automatically detecting physical presence and confirming one's identity through pattern recognition, computer vision and image analysis.

One of the Biometric attributes considered is aging. Aging is caused because of many reasons like DNA change, metabolism changes, UV rays from Sun, variation in facial tissues, Restructuring of facial bones etc. Face recognition system are adversely affected by the aging of the face. This idea plays huge role for new research area to be probed in the field computer vision. The age estimation is just carried out extensively to find out some patterns and variations as well as to get the best possible way to find out the various characteristic's that should be thought of. Another attribute is gender. Automatic gender classification is an important for many applications like surveillance, targeted advertisement's etc. This is done to differentiate between male and female based on the features of humans.

This literature elaborates descriptive minutiae and comparisons that are performed by author on various aspects like age, gender, and race. Also, different methods for extracting features, classification, evaluation for significant knowledge of research. This helps the enthusiastic researchers to enrol with deep learning aspects in classifying age and gender through human facial images.

\section{FACIAL GENDER AND AGE ESTIMATION: APPLICATION AND CHALLENGE'S}

Automation has covered wide area in real-world application area. Similarly, age and gender estimation can also be devised automatically. Age and gender classification techniques can improve computer's perceptional and interactional capabilities. Various applications for age and gender classification includes human-computer interaction (HCI) can provide users appropriate and customized services based on gender and age ground. Electronic customer relationship management is an internet-based technology; here personal data is examined for all class of consumers without intruding data privacy. Major aim of automation for classify age and gender is to predict age and gender class for each customer based on their facial images taken through camera and for demographic data. Age and gender classification can provide knowledge to improve the user experience in gaming and phone application. It can also be used for the verification or authentication accuracies based on soft biometric techniques. Age classification can control entry to unwanted content in televisions and Internet from children. In employment like police, military and government employments require age estimation during recruitment and while retirement age. In security and surveillance restricting of children access from adult vending machines like (alcohol and cigarette) and adulting websites movies, and monitoring of fraud detection. It can play major role in recognition of lost people, also for recognition of old people for purposes of identification from its previous images.

Facial gender classification and age estimation have many challenges. Gender prediction is followed for two-class that can be male or female. It is easy for human but not for machine to classify gender easily. For gender classification may methods and models have been bullied based on additional information from hairstyle, body shape, clothing and facial features. While in age estimation, it is not feasible to predict authentic age as of now. So, age grouping is still utilized for age prediction using facial images. Moreover, number of good datasets for age estimation and gender 
classification are limited for conduction of intense research.

\section{CONVALUTIONAL NEURAL NETWORK}

$\mathrm{CNN}$ is an active architecture of approach of profound learning which takes input as multimedia like images, videos or various $2 \mathrm{D} / 3 \mathrm{D}$ data. It is evident that gender and age estimation on basis of facial features deals with images and video data. Thus, various researches done by the researcher's and scientist lies on facial gender and age estimation focuses on CNN models. CNN model consists of different weights for every hidden neuron expressed in mathematical expression of multi-dimensional matrix. Further image transfers through hidden layers of $\mathrm{CNN}$, dimensions of weights and matrix gets remodel after every convolutional layer. CNN frameworks are designed by a well-fixed combination of layers such as narrow layers, sub sample layers, and full layers. Concentrated layers serve as building block to CNN and it is used to perform the basic function of convolution. Sub-sample layers perform major function for controlling over fitting issues by reducing the parameters and size by using max operation for maximum pooling. At last the fully connected layer of neurons is maps to all activation function of previous layers. There exists an additional RELU layer that helps in implementing the nonpurity function and correction in CNNs. The last layer is usually utilized as softmax layer that provides decimal feasibility to each output neuron. Researchers have proposed several interactive $\mathrm{CNN}$ architectures for basic neural network concept.

Predefined CNN architectures were investigated for this research keeping into account the recognition of gender as well as the estimation of the age from facial images, such as: VGG19 and VGGFace, ResNet, AlexNet and GoogLeNet.

\subsection{VGG19 AND VGGFace}

VGG is a deep CNN architecture designed by Karen Simonyan and Andrew Zisserman at Oxford. VGG19 consist of 19 weight layers in the network and so named as VGG19. In 2014 the VGG16 famed in the ImageNet competition as most accurate architecture and was correlate with InceptionNet. However, in some instances the additional hidden layer of VGG19 was advantaged to get more accurate findings than VGG16. Basic VGG architecture consists of layers that each have maximum pooling layers. Two fully associated layers are comprising under every VGG designs having size of 4096 with the dropout of $50 \%$, along with a size 1000 layer of softmax.

\subsection{ResNet}

ResNet is an abbreviation for Residual Network. ResNet introduced shortcut connections within layers to enable crosslayer connectivity, which are data independent and parameter free compared to the gates of the highway network. In highway networks, the non-residual functions are observed from layers when a gated shortcut is shutdown. ResNet-152, ResNet-50, ResNet-101, ResNet-34 and ResNet-18 are examined till date. In the ILSVRC 2015 it won top spot competition of classification with a top- 5 rate of error of $3.57 \%$.

\subsection{AlexNet}

In 2012 Alex Keyjevski design a neural network and therefore known as AlexNet. It is a combination of 8 layers in total. 5 of them are layered layers. Max-pooling layers are followed by some of these concentric layers. The final three layers are completely interconnected and known as fully connected layers. A ReLU layer is connected behind fully connected layers. Overfitting issues is controlled through dropout layer which is usually connected after the fully connected layer. AlexNet noted $16.4 \%$ for top-5 error.

\subsection{GoogleNet}

GoogLeNet is designed by inter-connecting nine Inception modules to recommend a deeper architecture. As a part of basic CNN architecture, Maximum pooling layers that helps in reducing the parameter size follows some inception modules for GoogLeNet architecture. Unlike Alex-Net and VGG-Net architecture, GoogLeNet does not consider a firm Convolution size. Generally, sizes of convolutional kernels are $1 \times 1,3 \times 3$ and $5 \times 5$. The $1 \times 1$ convolution layers implements feature dimensionality reduction function that is processed to broad network. GoogLeNet exceed AlexNet and VGGNet as conclusion to top- 5 error that reduces to $6.67 \%$ by using 22 convolutional layers.

\section{FACIAL GENDER CLASSIFICA- TION AND AGE ESTIMATION BASED ON NEURAL NETWORK}

Brief summary of research covering each individual neural net based facial characteristics, gender and age estimation, examined by the researchers between 2015 to 2019 is given bellow.

\subsection{CNN [Gil Levi et al.]}

Author has recommended CNN architecture for classifying gender and age using benchmark database of adience facial images with a better performance. The architecture is built up of one output layer and three fully connected layers. A part of starting two layers of convolutional layers contains local response normalization layer and connected to repaired linear operation (ReLU) along with the max-pooling layer for each convolutional layer. Smaller network design has been introduced to reduce over fitting problem. Every entirely associated layer encloses 512 neurons all. Output of the neural network is depending upon SoftMax layer for which input is provided through output of previous entirely associated layer. Cropped and over sampled facial mugshots where used for gender and age estimation. Author noted Mean accuracy \pm Standard error for age as well as the gender, where classification of the age using misperception matrix. Literal efficiency of single cropped image for estimating age results to $49.5 \pm 4.4$ and for 1 -OFF the efficiency results to $84.6 \pm$ 1.7. Whereas, over-sample method results efficiency of $50.7 \pm$ 5.1 and 1-OFF efficiency of $84.7 \pm 2.2$.

\subsection{CNN with Multiple Output}

[Zhenxing Niu et al.] developed a numerous output convolutional neural network for solving various classification problems. Collective binary classification problems are secured through an ordinal regression issue. For both MORPH and AFAD dataset results have been appraised as expected. For feature extraction module biologically, inspired features can be accessed and undergoes back propagation procedure. Proposed architecture works on three convolution layers, two Pool layers, 3 normalization layers along with a single fully connected layer. Metric along with the ordinal regression methods are correlated and acknowledged for both datasets by applying MAE metrics that results to 3.27 and 3.34 estimation on datasets respectively for propose research. These values can be used for any data specific schemes like colour images. Best results have been achieved from Ordinal Regression CNN (OR-CNN) and study of Cumulative score in a graphical manner. Authors have also tested the approach model on 
grayscale images versus colour images.

\subsection{Deep RoR Architecture}

[Ke Zhang et al.] offered an architecture of $\mathrm{CNN}$ in case of age and gender classification annotated on purchase residual of residual networks (RoR), that optimizes accurate gender and age estimation. To enhance the accuracy and solve overfitting issue, RoR gets qualified on ImageNet likewise then further customize on the IMDB-WIKI-101 dataset (Subsequently cleaning, dataset is separated in 101 classes based on age and named it IMDB-WIKI-101 dataset) for analysing the facial features of image. And later transformed into adience dataset. They used 64, 128, 256 and 512 filters successively under the conversational layers, also filter of each type has diverse number (L1, L2, L3, L4, correspondingly) of elementary blocks that form four elementary block groups.

\subsection{An Optimized CNN Architecture}

[M. Fatih Aydogdu et al.] offered architecture for age assessment known as an enhanced Convolutional Neural Network. The framework which is CNN-based can be justified with the ratios of training times, success-error along with success rate's standard deviations of; expending exact, top-3 and 1-off criterion based on the strength of the age estimation results. Architecture implies 4 convolutional as well as 2 completely associated layers which can be above as correlated with other architectures which are CNN-based along various sequence of layers. Technique has been evaluated on more than 55,000 images of face database. Excluding the introductory convolutional layer, every layer is pursued by Rectified Linear Unit (ReLu) layers, Maximum Pooling layers, as well as the Normalization (Norm) layers. With linking respective of the 4 stages of convolution with every 4 of the stage which is completely connected, overall, to the 16 architectures of $\mathrm{CNN}$ were formed mentioned above. With exact success percentage of CNN with Mean of 46.39 and STD of 27.35.

\subsection{Ranking CNN}

[Shixing Chen et al.] proposes a framework that generates binary output for sub networks that at last combines to obtain the age labels for age classification by giving input facial images. Independently, features were learned during designing all array of age class. Due to which various age class patterns were discovered and leads to estimated evaluation. To reduce over fitting labelled data was used and each age group was trained individually. For ranking of ages, authors have provided a tighter error constraint that is average analysis of all errors evaluated by classifiers. Three theorems were proven in this research paper. One minimization of binary error is that it can cut out final error of the classifier. Secondly ranking CNN and SoftMax are highly correlated. Thirdly a distinct upper bound defines precise error for ranking CNN. Model was trained on MORPH dataset and defined as accuracy, mean absolute error, cumulative score along with Tset consequences. Ranking CNN estimates evaluation of $89.90 \%$ for $\mathrm{L}=6$ and $92.93 \%$ for $\mathrm{L}=7$ and for mean absolute error score of 2.96 .

\subsection{Feedforward attention for age and gender recognition}

[Pau Rodriguex et al.] (i) An attention CNN ("where") that expects the finest grid of attention to display a glimpse, (ii) a patch CNN ("what") which is predicted by the attention grid created on their significance, Evaluates patches at high resolution along with (iii) a multi-layer perceptron (MLP) which incorporates statistics derived from CNNs which then achieves final classification. Given here are the three basic modules considered in proposed model. They explained modules in further detail. They used the VGG16 model as it became the most known for high accuracy that backs standard $\mathrm{CNN}$ in most intensive classifying frameworks, although can be studied on another CNN. They both also consider two strategies to merge CNN's feature maps: (i) compress them after L2 becomes normal, and (ii) study estimation of patched $\mathrm{CNN}$ for various feature maps and for attention CNN the feature map space and combine them.

\subsection{Wide CNN}

[Sepidehsadat Hosseini et al.] approached a wide CNN model for joint age-gender estimation, with help of Gabor filter return value as input. Its responses are become skilled at by back propagation in an end-to-end structure. The orientations of Gabor filter result are impeccably coordinated with experience lines consequently it will make a lot at ease for the networking to concentrate over them. To keep the excess statistics that is received as of the Gabor filters together to the first representation, the prejudiced amount of image as well as responses of the Gabor is actually utilized when the feedback towards CNN. It's mentioned that the perfect weights may be completely altered for various kinds of pictures. The networking is actually applied on Nvidia GeForce GTX 1060 6G 192 GPU along with it has taken approximately 100 minutes to receive the outcome (10000 Iteration). With boost up to $7 \%$ p as part of age-accuracy along with $2 \%$ p in gender reliability as associated to the state-of-the art form strategies.

\subsection{LMTCNN (Lightweight Multi-Task)}

[Jia-Hong Lee et al.] They presented a powerful CNN known as lightweight multi-task CNN for age assessment along with the gender acknowledgement. To trim model size as well as including the time of inference in depth-wise divisible convolution is utilized by Lightweight multi-task convolutional neural network which were critical for mobile applications. For feature extraction only one $\mathrm{CNN}$ is used and as well as for various function in their system. The point wise convolution is a convolution has $1 \times 1$ kernel's size, as well as it associates the standards of output in depth-wise convolution. It composed of one common layer of convolution, two depth-wise separable layers of convolution along with 2 fully associated layers. Network was implemented using the Tensorflow framework on their machine by means of Intel Xeon E5 3.5 GHz CPU, 64G RAM and GeForce GTX TITAN X GPU.

\subsection{Conditional Multitask learning (CMT)}

[ByungIn Yoo et al.] Basically incorporated architecture which predicts definite age of an individual centred on gender anticipation is known as Conditional Multitask (CMT) learning. For the conversion of definite labels of the age group into distinct value of the age labels it includes weak label expansion in it. All the efforts are carried out on FG-Net and MORPH-II repository. Comparisons as variation amid single undertaking, multitask along with CMT learning, qualitative studies, comparability with various CNN architectures and individuals along with prior tactics. This particular proposed technique has outperformed each of the state-of-the-art era opinion models of phone. As per the CASIA WebFace dataset, there suggested strategy accomplishes a MAE of 3.04 for cross validation process of fivefold along with a MAE of 3.08 for twofold cross validation protocol. On FG NET repository, the strategy accomplishes a MAE of 3.46. On Morph II repository, there suggested strategy accomplishes a 
MAE of 2.89 for cross validation process of fivefold along with a MAE of 2.91 for the protocol of twofold cross confirmation.

\subsection{Hybrid architecture (CNN -ELM (Extreme Learning Machine))}

[Mingxing Duan et al.] Features extraction is performed utilizing ELM and CNN is actually used for the goal of outcome distinction. The writers offer information of level and parameter choice developing, actions followed to stop over fitting, as well as detailed derivation of the backpropagation activity. Adience as well as the MORPH data source is utilized to get outcomes that are predictable. Experimental outcomes have proved its validity of particular hierarchical hybrid type for lots of real time uses. Just earlier detailing the suggested perform, the experts explain ELM as well as CNN in information for readers' lucidity. Crossbreed CNN ELM structure features a fair plan for developing constant level, difference normalization level, maximum pooling level as well as ELM distinction layer. The concentric level does convocation with the prior levels \& consequently extracts capabilities of interest. Distinctions normalization enables the calculation of various characteristic maps in the exact identical spatial place through utilizing level removal as well as segmentation activities. Max-pooling levels toss everything irrelevant information and just include information that is crucial. Lastly, the outputs of the total interconnection level are actually attached as inputs to the ELM as well as distinction effort are achieved. CNN ELM processing is completed as a program stage as well as a distinction stage. Error rates age as well as the reliability along with the gender abortions is actually the parameters utilized to confirm the improved functionality of given particular structure as of state-of-the-art criteria. For the era distinction, the reliability is actually discovered to be $52.3 \%$ and MAE 3.44.

\subsection{Soft Stagewise Regression Network (SSR-Net)}

[Tsun-Yi Yang et al.] A novel CNN unit recognized as Soft Stagewise Regression Network (SSR Net) for the estimation of age from an individual facial image. They deal with age approximation by carrying out multi class ordering also then reversing outcomes into reversion through computing the anticipated values. Each and every phase is actually liable for refining the determination of the prior stage of it's for precise age estimation. The model composes of the convolution of $3 \times 3$, normalization of the batch, non-linear stimulation and $2 \times 2$ pooling. The model of subsequent SSR-Net is quite solid as well as the receipts only $0.32 \mathrm{MB}$.

\section{OTHER METHODS FOR FACIAL GENDER CLASSIFICATION AND AGE ESTIMATION}

This section contains a brief summary of other techniques like Classification, regression and hybrid methods used for gender recognition and age classification. Other than above all models of neural nets for gender recognition and age classification. Survey has provided a superior option's for gender recognition and age classification for all methods which will lead to better and wide understanding.

[Michal Uricar et al.] proposed controlled production of SVM for prophesying gender and age from a facial image using deep features exploited from DEX. Since for

gender they used a binary SVM classifier and they trained 10 dissimilar splitting of the dataset of ChaLearn LAP 2016 in various multi-class SO-SVM classifier varieties for the estimation of probable age. Each multiclass SO-SVM predictor need a value of SoftMax normal refinement. Author terminates the finest is to associate the illustration capability in terms of the deep structures through the robustness capability of SO-SVM model for prophecy.

[F. Dornaika et al.] For training and testing phase regression tool were used in both phases. Thus, they also sympathetic in efficient shallow regressors. They used PLS (Partial Least Square) regressor. It's a statistical method that can discover relationship between groups of observed variables $\mathrm{X}$ and $\mathrm{Y}$ whereas $\mathrm{X}$ shows the observation and $\mathrm{Y}$ associated response. PLS is a powerful statistical approach which can achieve dimensionality reduction and classification/regression similar to PCA. Which will reduce the dimensionality of dataset?

\section{DETAILED DISCUSSIONS}

Literature undergoes thorough inquiry of facial gender and age classification using neural nets have been done. All the work comes under this domain has been noted and discussed. For all the interested researchers it provides a summary of human facial gender classification and age estimation using neural network. Various research works placed under neural nets are reviewed from various condition and technicalities like different type of models, dataset used, main inputs, evaluation metric and results are presented in tabular form. Other methods for gender classification and age estimation have been discussed in the section above for better understanding.

Table 1. Literature Survey

\begin{tabular}{|c|c|c|c|c|c|c|c|}
\hline $\begin{array}{c}\text { Sr } \\
\text { No. }\end{array}$ & References & $\begin{array}{c}\text { Year of } \\
\text { Publication }\end{array}$ & Models & $\begin{array}{c}\text { Dataset } \\
\text { Used }\end{array}$ & Main Inputs & $\begin{array}{c}\text { Evaluation } \\
\text { Metric }\end{array}$ & Results \\
\hline 1 & $\begin{array}{l}\text { Philip Smith } \\
\text { et al. [1] }\end{array}$ & 2018 & $\begin{array}{l}\text { Transfer } \\
\text { learning is } \\
\text { detected } \\
\text { using VGG- } \\
19 \text { and } \\
\text { VGG-Face }\end{array}$ & MORPH-II & & $\begin{array}{c}\text { MAE } \\
\text { Accuracy } \\
\%\end{array}$ & $\begin{array}{c}\text { MAE achieved } \\
4.10 \text { years which } \\
\text { improved the age } \\
\text { estimation model. } \\
\text { VGG19 achieved } \\
96 \%\end{array}$ \\
\hline 2 & $\begin{array}{c}\text { Ke Zhang et } \\
\text { al. [2] }\end{array}$ & 2017 & $\begin{array}{c}\text { Deep RoR } \\
\text { Architecture }\end{array}$ & $\begin{array}{l}\text { IMDB-WIKI } \\
\text { ImageNet }\end{array}$ & $\begin{array}{l}\text { The } 1000 \text { class } \\
\text { predefined } \\
\text { layer is } \\
\text { replaced by } \\
\text { ImageNet with } \\
\text { a prediction }\end{array}$ & $\begin{array}{c}\text { Accuracy } \\
\% \\
1-\text { off } \\
\text { accuracy }\end{array}$ & $\begin{array}{c}\text { The two-system } \\
\text { RoR-34 + IMDB- } \\
\text { WIKI achieved } \\
66 \% \text { on single- } \\
\text { model } \\
97 \% \text { on } 1 \text {-off }\end{array}$ \\
\hline
\end{tabular}




\begin{tabular}{|c|c|c|c|c|c|c|c|}
\hline & & & & & $\begin{array}{l}\text { layer of } 101 \\
\text { classes for age } \\
\text { prediction. }\end{array}$ & Error Rate & accuracy. \\
\hline 3 & $\begin{array}{c}\text { Sepidehsadat } \\
\text { Hosseini et } \\
\text { al. [3] }\end{array}$ & 2018 & $\begin{array}{l}\text { Wide CNN } \\
\text { Gabor Filter }\end{array}$ & Adience & $\begin{array}{c}\text { Input image } \\
\text { shaped to } \\
227 \times 227 .\end{array}$ & $\begin{array}{c}\text { Accuracy } \\
\%\end{array}$ & $\begin{array}{c}\text { Showing age and } \\
\text { gender-accuracy } \\
\text { of } 61 \% \text { and } 88 \% \\
\text { respectively. }\end{array}$ \\
\hline 4 & $\begin{array}{c}\text { Jia-Hong } \\
\text { Lee et al. [4] }\end{array}$ & 2018 & $\begin{array}{c}\text { Lightweight } \\
\text { Multi-task } \\
\text { CNN } \\
\text { (LMTCNN) }\end{array}$ & Adience & $\begin{array}{l}\text { New network } \\
\text { was introduced } \\
\text { LMTCNN. }\end{array}$ & $\begin{array}{c}\text { Training } \\
\text { Validation } \\
\text { Accuracy\% }\end{array}$ & $\begin{array}{l}\text { Showing age and } \\
\text { gender accuracy } \\
\text { of } 44 \% \text { and } 85 \% \\
\text { It can be realized } \\
\text { on mobile } \\
\text { devices with } \\
\text { limited } \\
\text { computational } \\
\text { resources. }\end{array}$ \\
\hline 5 & $\begin{array}{l}\text { Gil Levi et } \\
\text { al. [5] }\end{array}$ & 2015 & Deep CNN & Adience & $\begin{array}{l}\text { The new CNN } \\
\text { model was } \\
\text { proposed for } \\
\text { gender and age } \\
\text { classification. }\end{array}$ & $\begin{array}{c}\text { Confusion } \\
\text { Matrix } \\
\text { Accuracy } \\
\% \\
\text { Standard } \\
\text { error }\end{array}$ & $\begin{array}{c}\text { The best gender } \\
\text { accuracy for } \\
\text { proposed method } \\
86 \% \\
\text { The age best } \\
\text { accuracy for } \\
\text { proposed method } \\
85 \% \\
\text { Able to improve } \\
\text { results beyond } \\
\text { more training } \\
\text { data reports. }\end{array}$ \\
\hline 6 & $\begin{array}{c}\text { Nisha } \\
\text { Srinivas et } \\
\text { al. [6] }\end{array}$ & 2017 & $\begin{array}{l}\text { CNN with a } \\
\text { single } \\
\text { branch } \\
\text { architecture } \\
\text { CNN with } \\
\text { multiple } \\
\text { branches } \\
\text { architecture }\end{array}$ & $\begin{array}{l}\text { Wild East- } \\
\text { Asian Face } \\
\text { Dataset } \\
\text { (WEAFD) }\end{array}$ & $\begin{array}{c}\text { Full Face } \\
\text { Region } \\
\text { Experiment. } \\
\text { Use of hand- } \\
\text { selected face } \\
\text { subfields. }\end{array}$ & $\begin{array}{c}\text { Confusion } \\
\text { Matrix } \\
\text { Accuracy } \\
\%\end{array}$ & $\begin{array}{c}\text { Models of } \\
\text { ethnicity and age } \\
\text { were lacking in } \\
\text { training data. } \\
\text { Accuracy results } \\
\text { for age, gender, } \\
\text { and exact } \\
\text { ethnicity are } \\
38 \%, 88 \%, 33 \% \text {. }\end{array}$ \\
\hline 7 & $\begin{array}{c}\text { Michal } \\
\text { Uricar et al. } \\
{[7]}\end{array}$ & 2016 & $\begin{array}{c}\text { SO-SVM } \\
\text { Caffe } \\
\text { Framework }\end{array}$ & $\begin{array}{c}\text { ChaLearnLAP } \\
2016\end{array}$ & & $\begin{array}{c}\text { MAE } \\
\text { Accuracy } \\
\% \\
€ \text { Error }\end{array}$ & $\begin{array}{c}\text { E-error was } \\
\text { found with } 0.209 \\
\text { and MAE } 2.5 \\
\text { with an accuracy } \\
\text { of } 67 \% \text {. }\end{array}$ \\
\hline 8 & $\begin{array}{l}\text { M. Fatih } \\
\text { Aydogdu et } \\
\text { al. [8] }\end{array}$ & 2017 & $\begin{array}{c}\text { An } \\
\text { Optimized } \\
\text { CNN } \\
\text { architecture }\end{array}$ & MORPH & $\begin{array}{l}\text { A total of } 16 \\
\text { CNN } \\
\text { architectures } \\
\text { hunt } 4 \\
\text { complete stages } \\
\text { with each of } \\
\text { the } 4 \text { complete } \\
\text { stages. } \\
\text { Performed } 70 \\
\text { epochs. }\end{array}$ & $\begin{array}{c}\text { Mean } \\
\text { Standard } \\
\text { deviation }\end{array}$ & $\begin{array}{c}\text { Mean } 46.39 \% \\
\text { and exact } \\
\text { success\% of STD } \\
\text { with } 27.35 \% \text {. }\end{array}$ \\
\hline 9 & $\begin{array}{c}\text { ByungIn } \\
\text { Yoo et al. } \\
{[9]}\end{array}$ & 2018 & $\begin{array}{c}\text { CMT } \\
\text { Learning }\end{array}$ & $\begin{array}{c}\text { MORPH-II } \\
\text { FG-NET } \\
\text { CASIA- } \\
\text { WebFace }\end{array}$ & & $\begin{array}{c}\text { Mean } \\
\text { Label } \\
\text { Expansion }\end{array}$ & $\begin{array}{c}\text { With Mean of } \\
3.04\end{array}$ \\
\hline
\end{tabular}




\begin{tabular}{|c|c|c|c|c|c|c|c|}
\hline 10 & $\begin{array}{c}\text { Abhijit Das } \\
\text { et al. [10] }\end{array}$ & 2019 & $\begin{array}{c}\text { FaceNet } \\
\text { ResNet } \\
\text { (Version 1) }\end{array}$ & $\begin{array}{c}\text { UTKFace } \\
\text { BEFA }\end{array}$ & $\begin{array}{c}\text { Proposed } \\
\text { multi-task } \\
\text { CNN approach. } \\
\text { It was ranked } \\
\text { first in BEFA- } \\
\text { challenge of } \\
\text { European } \\
\text { Conference on } \\
\text { Computer } \\
\text { Vision (ECCV) } \\
\text { 2018. }\end{array}$ & $\begin{array}{c}\text { Accuracy } \\
\% \\
\text { Confusion } \\
\text { Matrix } \\
\text { Mean }\end{array}$ & $\begin{array}{l}\text { Accuracy for race } \\
84 \% \text {, gender } 94 \% \\
\text { and age } 72 \% \text {. }\end{array}$ \\
\hline 11 & $\begin{array}{c}\text { M. Del Coco } \\
\text { et al. [11] }\end{array}$ & 2017 & $\begin{array}{l}\text { Multi- } \\
\text { branch } \\
\text { CNN }\end{array}$ & Adience & $\begin{array}{l}\text { Proposed } \\
\text { network } \\
\text { consists of two } \\
\text { points: - } \\
\text { A depth } \\
\text { reduced } \\
\text { network. } \\
\text { A multi-branch } \\
\text { architecture. }\end{array}$ & $\begin{array}{c}\text { K-fold } \\
\text { validation } \\
\text { Accuracy } \\
\% \\
1 \text { class-off } \\
\text { accuracy \% } \\
\text { Confusion } \\
\text { Matrix }\end{array}$ & $\begin{array}{c}\text { Approach } \\
\text { proposed gets } \\
\text { accuracy of } 96 \% \text {. } \\
\text { With increase in } \\
\text { average accuracy } \\
\text { upto } 4.15 \% \text { for 1- } \\
\text { off estimation. }\end{array}$ \\
\hline 12 & $\begin{array}{l}\text { F. Dornalka } \\
\text { et al. [12] }\end{array}$ & 2018 & $\begin{array}{l}\text { Regression } \\
\text { Module } \\
\text { Transfer } \\
\text { Learning }\end{array}$ & $\begin{array}{c}\text { MORPH-II } \\
\text { Productive } \\
\text { Aging Lab } \\
\text { Face (PAL) } \\
\text { Chalearn2016 }\end{array}$ & $\begin{array}{l}\text { Considered five } \\
\text { deep features } \\
\text { by DCNN and } \\
\text { three shallow } \\
\text { image features. }\end{array}$ & Mean & $\begin{array}{c}\text { Chalearn } 2016 \\
\text { face images, } \\
\text { Ranging MAE } \\
\text { from } 9 \text { to } 11.9 \\
\text { years. } \\
\text { Accuracy } \\
\text { obtained by } \\
\text { transfer learning } \\
\text { can be correlated. }\end{array}$ \\
\hline 13 & $\begin{array}{c}\text { Jin Huang et } \\
\text { al. [13] }\end{array}$ & 2016 & $\begin{array}{l}\text { SVM and } \\
\text { KNN } \\
\text { Classifiers }\end{array}$ & $\begin{array}{l}\text { FG-NET } \\
\text { MORPH } \\
\text { Adience }\end{array}$ & $\begin{array}{c}\text { They } \\
\text { Constrained } \\
\text { database } \\
\text { (FG- } \\
\text { NET+MORPH) } \\
\text { and } \\
\text { unconstrained } \\
\text { database } \\
\text { (Adience). }\end{array}$ & $\begin{array}{l}\text { Confusion } \\
\text { Matrix } \\
\text { Accuracy } \\
\%\end{array}$ & $\begin{array}{l}\text { Accuracy for FG- } \\
\text { NET+MORPH } \\
\text { with SVM } \\
\text { method is } 93 \% \\
\text { and KNN is } 94 \% \\
\text { Accuracy for } \\
\text { Adience with } \\
\text { SVM method is } \\
50.7 \% \text { and KNN } \\
\text { is } 51.1 \%\end{array}$ \\
\hline 14 & $\begin{array}{l}\text { Jian Lin et } \\
\text { al. [14] }\end{array}$ & 2017 & VGG-16 & $\begin{array}{l}\text { FG-NET } \\
\text { Adience }\end{array}$ & $\begin{array}{l}\text { They adapted } \\
\text { Transform } \\
\text { learning in } \\
\text { unconstrained } \\
\text { age estimation. }\end{array}$ & $\begin{array}{c}\text { Accuracy } \\
\%\end{array}$ & $\begin{array}{l}\text { Without AvgOut- } \\
\text { FC Layer model } \\
\text { appearance } 55 \% \\
\text { and } 57 \% \\
\text { With AvgOut-FC } \\
\text { Layer model } \\
\text { display } 2 \% \text { better. }\end{array}$ \\
\hline 15 & $\begin{array}{c}\text { Ajita Rattani } \\
\text { et al. [15] }\end{array}$ & 2017 & $\mathrm{CNN}$ & Adience & $\begin{array}{l}\text { Proposed six- } \\
\text { layer CNN } \\
\text { model. }\end{array}$ & $\begin{array}{c}\text { Accuracy } \\
\% \\
1-\text { off } \\
\text { Accuracy } \\
\%\end{array}$ & $\begin{array}{l}\text { Reporting exact } \\
\text { and 1-off } \\
\text { accuracies are } \\
50 \% \text { and } 85 \% \\
\text { respectively. }\end{array}$ \\
\hline
\end{tabular}

Research work completed using CNN models are Deep RoR Architecture (Ke Zhang et al. 2017), Wide CNN with Gabor Filter (Sepidehsadat Hosseini et al. 2018), (LMTCNN) Lightweight Multi-task CNN (Jia-Hong Lee et al. 2018), FaceNet and ResNet (Abhijit Das et al. 2017), VGG19 and VGGFace (Philip Smith et al. 2018), VGG-16 (Jian Lin et al.
2017), CNN Multiple Output (Zhenxing Niu et al. 2016), Ranking CNN (Shixing Chen et al. 2017), (CMT) Conditional Multitask Learning (ByungIn Yoo et al. 2018) and (CNNELM) Convolutional neural network -extreme learning machine (Mingxing Duan et al. 2018). It's well worth noting that the most popular datasets for gender as well as years 
distinction analysis are actually Adience datasets and MORPH. MORPH can be obtained to public within Creative Commons (CC) license along with it consists of 2 albums. Album one includes of 515 digital pictures as well as 55,134 digital pictures of 13,000 individuals is contained by album two. These pictures are actually taken during various spans of occasions to get age variants as well as album two is currently growing. The majority of the analysis functions in Table 1 has utilized these 2 directories for the evaluations of theirs. Adience can be obtained to public within Creative Commons (CC) license and 26,580 pictures of 2284 various people are contained by it. FG-NET is yet another publicly understood era repository that can be used in recommendations as ByungIn Yoo et al. (2018), Jin Huang et al. (2016) and Jian Lin et al. (2017). FG-NET collection comprises of experience pictures from various ages of various topics. It consists of 1002 pictures of eighty-two various individuals. Grow old variants of that data source range from zero to sixty-nine years old individuals for experimental succeeds on age, gender as well as man recognition. A number of recommendations have implemented a few various other purposeful directories such as Asian Face Age

(WEAFD) wild East-Asian Face Dataset (Nisha Srinivas et al. 2017), IMDB-WIKI (Ke Zhang et al. 2017) and (Tsun-Yi Yang et al. 2018), MegaAge-Asian (Tsun-Yi Yang et al. 2018), UTKFace and BEFA (Abhijit Das et al. 2019), ChaLearnLAP 2016 (Michal Uricar et al. 2016) and (PAL) Productive Aging Lab Face (F. Dornalka et al. 2018).

\section{Datsets used in various References}

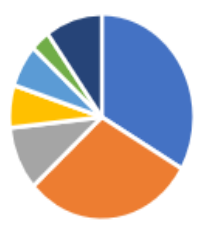

$$
\begin{array}{lll}
\text { " MORPH } & \text { " Adience } & \text { " FG-NET } \\
\text { " ChaLearnLAP 2016 } & \text { " IMBD-WIKI } & \text { " PAL } \\
\text { " Asian DataSet } & &
\end{array}
$$

\section{Fig 1: Datasets used in various references}

Explanation of the ratio of using of various older directories in various research documents working with a pie chart is actually displayed above in Fig.1 Optimum place is actually taken in place by the MORPH data source as well as bare minimum is actually taken up by PAL repository and several recently produced directories in various research documents as BEFA and UTKFace (Abhijit Das et al. 2019) along with Private Datasets of Asian.

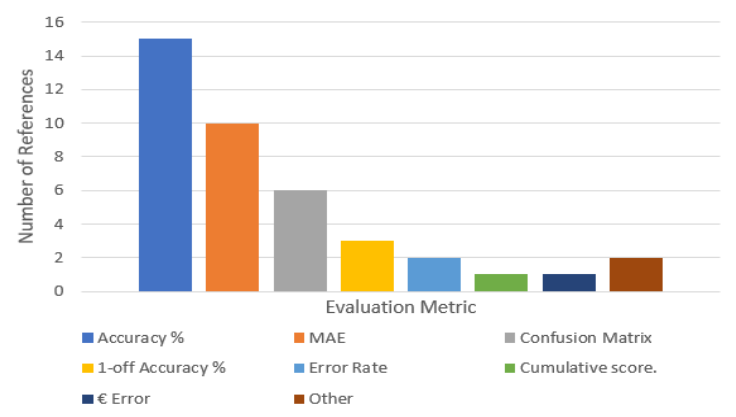

Fig 2: Evaluation Metric used in various references
Most of the references like Smith et al. Ke Zhang et al. (2017), Sepidehsadat Hosseini et al. (2018), Jia-Hong Lee et al. (2018), Gil Levi et al. (2015), Nisha Srinivas et al. (2017), Michal Uricar et al.(2016), Abhijit Das et al. (2019), M. Del Coco et al. (2017), Jin Huang et al. (2016), Jian Lin et al. (2017), Ajita Rattani et al. (2017), Shixing Chen et al. (2017), Mingxing Duan et al. (2018) and Pau Rodriguex et al. (2017) have used accuracy for the performance evaluations of their work. Accuracy can be achieved from the right sample of the population to regulate the precision and accurate the outcomes.

References similar to Smith et al. (2018), Michal Uricar et al. (2016), M. Fatih Aydogdu et al. (2017), ByungIn Yoo et al. (2018), Abhijit Das et al. (2019), F. Dornalka et al. (2018), Shixing Chen et al. (2017), Zhenxing Niu et al. (2016), Pau Rodriguex et al. (2017), Tsun-Yi Yang et al. (2018) have used Mean Absolute Error (MAE) as per the parameter of evaluation. The MAE formula is specified as:

$$
M A E=\frac{1}{N} \sum_{K=1}^{N}|a-\hat{a}|
$$

Right here $\mathrm{N}$ reports the selection of pictures tried in the testing, it adopts the particular era of the kth individual and ${ }^{\wedge} \mathrm{a}$ adopts the expected age of the kth individual. Reduced will be the MAE, much better is actually the overall testing performance.

References similar to confusion Gil Levi et al. (2015), Nisha Srinivas et al. (2017), Abhijit Das et al. (2019), M. Del Coco et al. (2017), Jin Huang et al. (2016), and Pau Rodriguex et al. (2017) matrix is also calculated by some researchers. Confusion matrix is in a tabular form utilised to define the classifier's performance by predicting values as positive or negative and actual values as false or true on a dataset of test to which the true values have previously delivered.

Reference like Shixing Chen et al. (2017): has implemented Cumulative Score (CS) for evaluation as experimentally. Collective Score may be clarified by the formulation provided as:

$$
C S(k)=\frac{N_{e \leq k}}{N} \times 100 \%
$$

Right here $\mathrm{N}$ once again describes the selection of images underneath test as well as $\mathrm{N}_{\mathrm{e} \leq \mathrm{k}}$ matter directs the number of examination pictures whose complete mistake involving the estimated and actual age is actually under $\mathrm{k}$ years.

\section{CONCLUSION AND FUTURE WORK}

Overall study of contribution made on gender classification and age estimation can be used in to solve the real-time application problems. In this paper most of the research work done is in Convolutional Neural Networks. Eleven unlike types of neural networks have been discussed with their MAE and accuracy obtained by models. Additionally, function extraction in addition to distinction of a few functions is actually carried out using just an individual element extractor or maybe a one-time classifier along with in various additional works, fusion is actually followed to do distinction process or maybe attribute extraction.

On the future direction, results that are Good for gender recognition as well as years opinion can continue to be received utilizing transfer learning strategies with expansion in reliability. Combos of fusions as well as datasets of attributes might be what is on the horizon for the development 
of rich learning and from 2D to 3D Facial Data. Moreover, ethnicity estimation, Affective behaviour analysis and numerous additional demographic features could be verified for the performance of them by the classifier of Neural Networks.

\section{ACKNOWLEDGEMENTS}

I would like to thank my guide Dr. Nitin N. Pise for his support in this survey. Also, Siddhivinayak Kulkarni Sir for helping me the best way possible.

\section{REFERENCES}

[1] Philip Smith, Cuixian Chen Transfer Learning with Deep CNNs for Gender Recognition and Age Estimation, IEEE International Conference on Big Data 2018.

[2] Ke Zhang, Liru Guo, Miao Sun, Xingfang Yuan, TonyX. Han, Zhenbing Zhao and Baogang Li Age Group and Gender Estimation in the Wild with Deep RoR Architecture, IEEE Access COMPUTER VISION BASED ON CHINESE CONFERENCE ON COMPUTER VISION Volume 5 (CCCV)2017.

[3] Sepidehsadat Hosseini, Seok Hee Lee, Hyuk Jin Kwon, Hyung Ii Koo and Nam Ik Cho Age and Gender Classification Using Wide Convolutional Neural Network and Gabor Filter, Institute for Information and communications Technology Promotion (IITP) 2018.

[4] Jia-Hong Lee, Yi-Ming Chan, Ting-Yen Chen and ChuSong Chen Joint Estimation of Age and Gender from Unconstrained Face Images using Lightweight Multi-task CNN for Mobile Applications, IEEE Conference on Multimedia Information Processing and Retrieval 2018.

[5] Gil Levi and Tal Hassner Age and Gender Classification using Convolutional Neural Networks, Intelligence Advanced Research Projects Activity (IARPA) 2015.

[6] Nisha Srinivas, Harleen Atwal, Derek C. Rose, Gayathri Mahalingam, Karl Ricanek Jr. and David S. Bolme, Age, Gender, and Fine-Grained Ethnicity Prediction using Convolutional Neural Networks for the East Asian Face Dataset, 12th International Conference on Automatic Face \& Gesture Recognition 2017.

[7] M Uricár, R Timofte, R Rothe, J Matas and L Van Gool Structured output svm prediction of apparent age, gender and smile from deep features, Proceedings of the IEEE Conference on Computer Vision and Pattern Recognition Workshops 2016.

[8] M. Fatih Aydogdu and M. Fatih Demirci Age Classification Using an Optimized CNN Architecture, Association for Computing Machinery 2017.

[9] ByungIn Yoo, Youngjun Kwak, Youngsung Kim, Changkyu Choi and Junmo Kim, Deep Facial Age Estimation Using Conditional Multitask Learning with Weak Label Expansion, SIGNAL PROCESSING LETTERS, VOL. 25, NO. 62018.

[10] Abhijit Das, Antitza Dantcheva and Francois Bremond Mitigating Bias in Gender, Age and Ethnicity Classification: A Multi-task Convolution Neural Network Approach, European Conference of Computer Vision (ECCV) 2019.
[11] Marco Del Coco, Pierluigi Carcagni, Marco Leo, Paolo Spagnolo, Pier Luigi Mazzeo and Cosimo Distante Multi-branch CNN for Multi-scale Age Estimation, Springer ICIAP 2017, Part II, pp. 234-244, 2017.

[12] F. Dornaika, Arganda-Carreras and C. Belver, Age estimation infacial images through transfer learning, Machine Vision and Applications 2018.

[13] Jin huang, Bin Li, Jia Zhu and Jian Chen, Age classification with deep learning face representation, Multimed Tools Appl, Science+Business Media New York 2017.

[14] Jian Lin, Tianyue Zheng, Yanbing Liao and Weihong Deng, CNN-Based Age Classification via Transfer Learning, Springer International Publishing AG 2017

[15] Ajita Rattani, Narsi Reddy and Reza Derakhshani, Convolutional Neural Network for Age Classification from Smart-phone based Ocular Images, IEEE International Joint Conference on Biometrics 2017.

[16] Shixing Chen, Caojin Zhang and Ming Dong, Deep Age Estimation: From Classification to Ranking, TRANSACTIONS ON MULTIMEDIA 2017.

[17] Mingxing Duan, Kenli Li, Canqun Yang and Keqin Li, A hybrid deep learning CNN-ELM for age and gender classification, Neurocomputing 275 (448-461) 2018.

[18] Zhenxing Niu, Mo Zhou, Le Wang, Xinbo gao and Gang Hua, Ordinal Regression with Multiple Output CNN for Age Estimation, Conference on Computer Vision and Pattern Recognition 2016.

[19] Pau Rodr'1guez, Guillem Cucurull, Josep M. Gonfaus, F. Xavier Roca, and Jordi Gonzale, Age and Gender Recognition in the Wild with Deep Attention, Pattern Recognition 2017.

[20] Tsun-Yi Yang, Yi-Hsuan Huang, Yen-Yu Lin, Pi-Cheng Hsiu and Yung-Yu Chaung, SSR-Net: A Compact Soft Stagewise Regression Network for Age Estimation, Proceedings of the Twenty-Seventh International Joint Conference on Artificial Intelligence 2018.

[21] Prachi Punyani, Rashmi Gupta and Ashwani Kumar, Neural networks for facial age estimation: a survey on recent advances, Artificial Intelligence Review Springer Nature B.V. 2019.

[22] Raphael Angulu, Jules R. Tapamo and Aderemi O. Adewumi, Age estimation via face images: a survey, Journal on Image and Video Processing 2018.

[23] Marwa Ahmed and Serestina Viriri, Deep Learning Using Bayesian Optimization for Facial Age Estimation, ICIAR 2019, pp. 243-254, 2019.

[24] Chang-Ling Ku, Chun-Hsiang Chiou, Zhe-Yuan Gao, Yun-Je Tsai and Chiou-Shann Fuh, Age and Gender Estimation Using Multiple-Image Features, CCBR 2013, pp. 441-448, 2013.

[25] Prajakta A. Melange and Dr. G. S. Sable, Age Group Estimation and Gender Recognition Using Face Features, Volume-7, Issue-7, PP 01-07 (The International Journal of Engineering and Science) 2018. 